\title{
Inkjet Printable Flexible Thin-Film NCQD Photodetectors on Unmodified Transparency Films
}

\author{
Jingda Wu and Lih Y. Lin, Fellow, IEEE
}

\begin{abstract}
We proposed and experimentally demonstrated a cost-efficient and easy-to-fabricate flexible thin-film nanocrystal quantum dot (NCQD) photodetector, by sandwiching a piece of NCQD-soaked tracing paper between two PEDOT:PSS electrodes. The highly conductive and transparent multilayer polymer electrodes are printed with an office inkjet printer without further patterning on commercial unmodified transparency films. The tracing paper is able to act as both the bed for NCQD attachment and the interspacing layer to prevent short-circuiting in vertical devices. Consistent increase in device performance is observed when the bending radius becomes smaller. Linear $I-V$ characteristics are obtained, which show our device structure works as a photoconductor.
\end{abstract}

Index Terms - Inkjet printing, quantum dots, thin film devices, photoconducting devices, flexible structures.

\section{INTRODUCTION}

$\mathbf{F}$ LEXIBLE device, which relies little on large manufacturing plant and is compatible with low-cost fabrication methods, has risen to be an important direction for optoelectronic research and industry. Its superb integratibility also drives the commercialization potential for wearable, foldable and roll-up devices. While organic materials currently dominate this field, nanocrystal quantum dot (NCQD) offers an alternative way with size-dependent excitation/emission wavelength tunability and high optical quality without sacrificing solution-processability. However, research on NCQD or other inorganic material-based flexible optoelectronic devices are still at its infancy, especially in the field of photodetectors.

PEDOT:PSS (poly (3,4-ethylenedioxythiophene) poly (styrenesulfonate)) has long been reported as a material for transparent electrodes with high conductance, high transparency, thermal and electrochemical stability [1]. PEDOT acts as the main conductive part, while PSS is the water-soluble polyelectrolyte to provide this material with solution-processability. Though ITO (indium tin oxide) has better performance in conductance and is still widely used in today's transparent optoelectronic devices, its flexibility [2] is limited and the cost remains high due to the inclusion of rare earth material indium and vacuum deposition method.

Manuscript received June 11, 2013; revised January 7, 2014; accepted February 10, 2014. Date of publication February 20, 2014; date of current version March 11, 2014. This work was supported in part by the National Science Foundation under Grant ECCS-0925378 and in part by the UW Commercialization Grant Fund.

The authors are with the Department of Electrical Engineering, University of Washington, Seattle, WA 98195 USA (e-mail: albuswu@uw.edu; lylin@uw.edu).

Color versions of one or more of the figures in this letter are available online at http://ieeexplore.iee.org.

Digital Object Identifier 10.1109/LPT.2014.2305983
PEDOT:PSS is promising in a way that it can be deposited and patterned easily with state-of-the-art printing techniques with decent performance and low cost.

Inkjet printing method has been exploited by many researchers to deposit solution-processable materials such as conductive polymers [3], [4], quantum dots [5], metal nanoparticles [6], carbon nanotubes [7], etc. Comparing with other fabrication methods such as spin-coating and thermal evaporation, inkjet printing offers a way to significantly lower the cost and material wastage while at the same time being able to deliver large-area and mask-less noncontact precise patterning at a high speed. While precise control of each droplet can be achieved with a complicated material printer like Dimatix DMP-2800, commercially available low-cost office inkjet printers [8] have been proved to be reliable for patterning well-controlled PEDOT:PSS films with little system modification. Moreover, the commonly used image processing software like Adobe Photoshop has the ability to precisely control printing patterns to a single pixel, which further facilitates the exploitation of office inkjet printer for material thin-film printing.

In terms of flexible substrate materials, paper has been broadly researched for applications in photovoltaics [9], photodetectors [10] and displays [11] because of their super-low cost and versatile flexibility. However, paper substrates suffer from low tolerance on wetting conditions and low resistance to strong mechanic forces. Furthermore, it's not straightforward to form continuous and smooth films on the paper surface for electrodes because of the fiber cellulose structure. Evaporation techniques [9] may be used for electrode deposition but add significantly more cost and complexity to the fabrication process. In view of all these problems, polyethylene terephthalate (PET) substrates outperform paper substantially with little increase in cost. The transparency film, commonly used for classroom presentations, is one of the most available and costefficient material among all kinds of PETs. Its transparency, mechanic and electrochemical durability, as well as high smoothness make it an ideal substrate for low-cost flexible optoelectronic devices.

Although paper may not be the best substrate material, we have discovered that its cellulose structure assists the attachment of NCQDs to form uniform photoconductive films which act as good inter-electrode spacing/structure layers for optoelectronic devices. The flexibility of the paper leads to better contacts between QDs and electrodes. Its insulating nature also helps preventing short-circuiting of the device.

In this letter, we report a simple method for flexible NCQD photoconductive detector fabrication on unmodified 


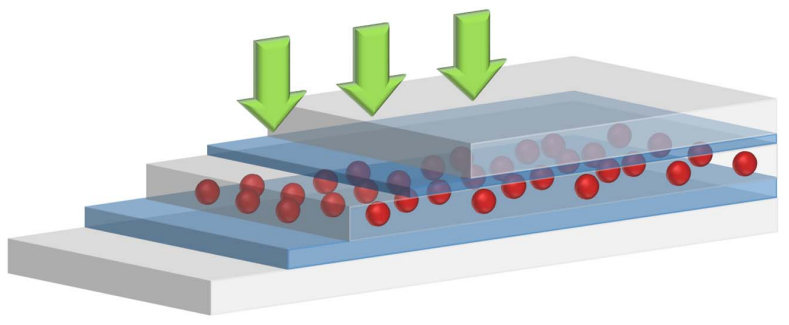

Fig. 1. Schematics of a flexible NCQD photoconductive detector. Materials for layers from top to bottom: transparency film/PEDOT:PSS/tracing paper (CdSe QDs)/PEDOT:PSS/transparency film. Light illumination is from the top.

transparency films, with inkjet-printed PEDOT:PSS electrodes and NCQD-saturated paper interspacing layer (Fig. 1). High precision multi-layer printing of the electrode is achieved simply by reprinting the pattern after the previous printed film is dried, and its electrical and optical properties are characterized. The device performance under different bending radii is also characterized and discussed.

\section{FABRICATION}

\section{A. Inkjet Printing of Electrodes}

PEDOT:PSS electrodes are printed with an HP1000 thermal office inkjet printer on an unmodified letter-size transparency film (PP2500 from 3M). Original HP61 black cartridge, which is compatible with the printer, is cleaned and without further modification. Patterns are precisely controlled with accuracy to a single pixel by Adobe Photoshop. Unlike the film thickness control method described in reference [8], we set the luminosity to be zero, i.e. the color seen on the computer screen is totally black, to obtain highest thickness and thus the best conductance. PEDOT:PSS ink (Sigma-Aldrich), $0.8 \%$ solids in water, is prepared with $0.1 \%$ (vol.) Triton-X 100 [8] as surfactant to prevent formation of "coffee rings" during printing and achieve good film uniformity. The formulation is injected into the cartridge with a syringe through a $0.45 \mu \mathrm{m}$ filter to filter out large particles to prevent nozzle clogging. Multi-layer printing is achieved simply by adding the printed films back to the printer paper holder and align with the edge of the paper holder, then repeat the printing process. The electrodes are baked under $80-100^{\circ} \mathrm{C}$ in ambient atmosphere for about 10 minutes to dry the solvents after each printing to avoid the damage that can be caused by the rolling system of the printer during next printing. Sub- $100 \mu \mathrm{m}$ alignment accuracy is achieved for multi-layer printing along the rolling direction. We discovered that slight misalignment in each printing actually helps to smoothen the film and improve conductivity due to the non-even polymer distribution in the formulation. After the whole printing cycle, the electrodes are annealed at the same temperature mentioned above for 2 hours or more to dry all the organic and water residues.

\section{B. Quantum Dot Deposition and Device Fabrication}

CdSe quantum dot (NN-LABS, Emission Peak at 630nm, $5.2-6.2 \mathrm{~nm}$ in size, suspended in toluene, -Octadecylamine), is used in the photodetector acting as the photoconductive

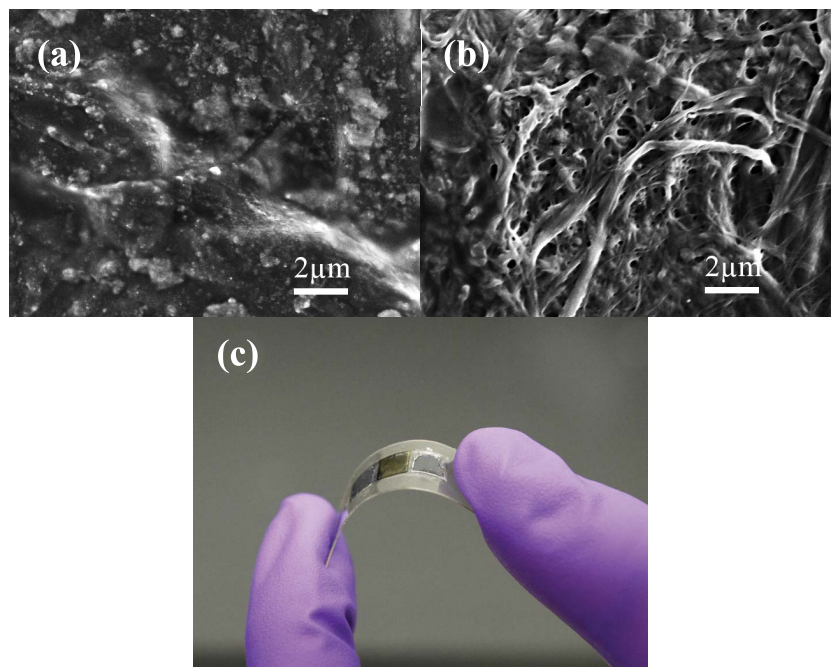

Fig. 2. SEM image of the fiber cellulose structure of tracing paper with (a) and without (b) CdSe QDs. (c) A flexible NCQD thin film photodetector under bending.

semiconductor material. In order to reduce the influence of ligands on photoconductivity, we washed the original NCQDs at least 3 times in toluene and ethanol mixture before deposition. Each wash is done with ethanol and subsequent centrifuging. The precipitations are redissolved by toluene for next wash or deposition. The prepared QDs are drop-casted onto aspurchased tracing paper (Roselle Paper, $\sim 35 \mu \mathrm{m}$ thick) instead of being coated on the electrodes directly. The optical transparency at 532nm for the tracing paper used in the experiments is $\sim 25 \%$. However, strong light scattering effect in the fibrous structure helps to enhance the light absorption of QDs. To maximize the QD density in the tracing paper to increase photoresponsivity of the device, multiple drops of QDs are deposited until sufficient density is obtained. SEM images for tracing paper cellulose structures with and without QDs are shown in Fig. 2(a) and (b). QDs are attached to the fibers and form continuous films along the fibers. The QD-soaked tracing paper is sandwiched between two PEDOT:PSS electrodes printed on transparencies after solution being dried and the device is laminated with glue. While it's not straightforward to obtain glue with both good conductivity and transparency to laminate the active layer, we apply glue at the periphery of the tracing paper on transparency films. Two flat metal plates are used to clamp the device until the glue's dried. An actual flexible device is shown in Fig. 2(c).

\section{Electrode ChARACTERIZATION}

Uniform polymer electrodes with areas of $5 \mathrm{~mm} \times 10 \mathrm{~mm}$ were printed with variation in layer quantities from 1 to 8 [Fig. 3(a)]. The transmittance of the printed film decreases when the layer number increases, which can be seen visually. The sheet resistance was measured by four-point probe technique and the results are shown with film transmittance under $532 \mathrm{~nm}$ light illumination in Fig. 3(b). Note that the transparency film itself contributes to $10 \%$ light loss (as can be seen visually in Fig. 3(a), comparing to the center part of 


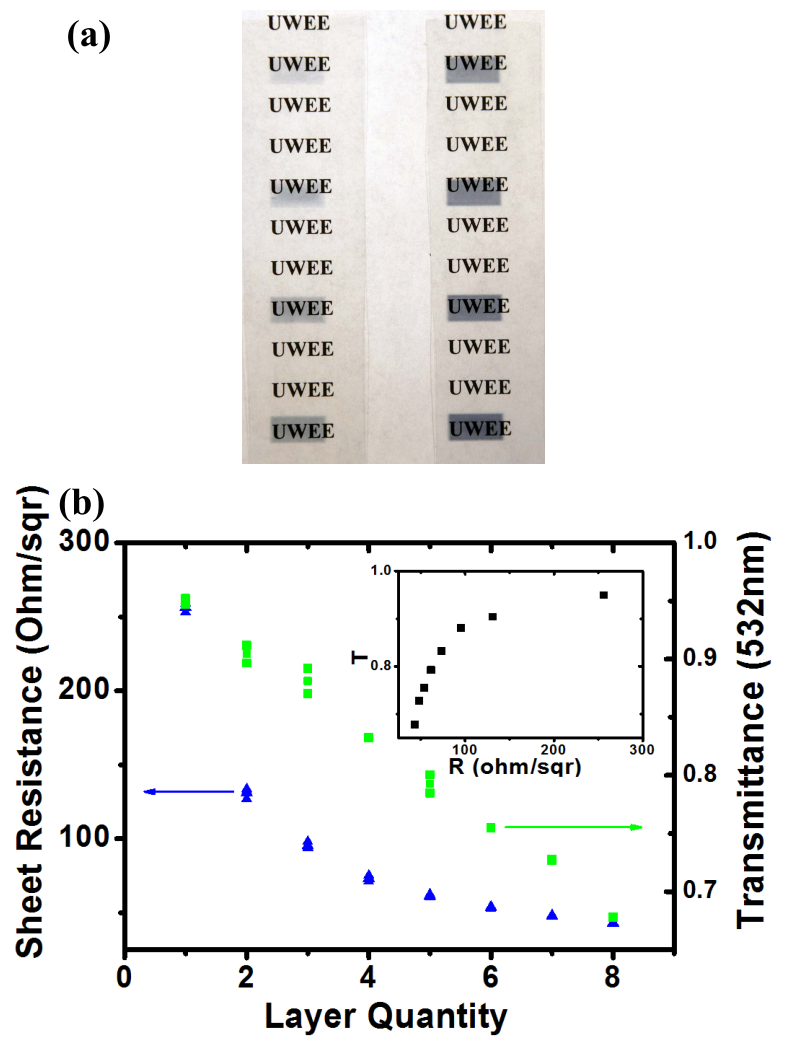

Fig. 3. (a) A photo of the electrodes with different number of layers on a white paper with texts; Layer quantities from top left to bottom left is 1 to 4 , and 5 to 8 for top right to bottom right. (b) Sheet resistance and transmittance for inkjet-printed PEDOT:PSS films as a function of the layer quantity. Multiple measurements were taken at different regions. Inset: transmittance vs. sheet resistance.

the paper without the transparency film) and the data shown in Fig. 3(b) is the net transmittance for the PEDOT:PSS film. Linear dependence of transmittance on layer number is obtained, while for resistance the value decreases hyperbolically with the layer number. It is because the multi-layer electrodes act as parallel resistors due to the baking after each printing.

The relation between film sheet resistance $R_{s h}$ and transmittance $\mathrm{T}$ is given by the following equation [12]:

$$
\mathrm{T}=\left(1+\frac{Z_{0}}{2 R_{s h}} \frac{\sigma_{o p}}{\sigma_{d c}}\right)^{-2},
$$

where $Z_{0}$ is the impedance of free space $(377 \Omega), \sigma_{o p}$ and $\sigma_{d c}$ are the optical and electrical conductivities, respectively. The value $\sigma_{d c} / \sigma_{o p}$ is normally used to evaluate the performance of transparent electrodes, and traditional metal oxide electrodes have $\sigma_{d c} / \sigma_{o p} \sim 35$. It's always important to have high transmittance and low sheet resistance, and thus it's better to have large $\sigma_{d c} / \sigma_{o p}$ value. For our PEDOT:PSS electrodes, the value obtained is $\sim 28$, which is slightly smaller than the metal oxide electrodes but acceptable. As shown

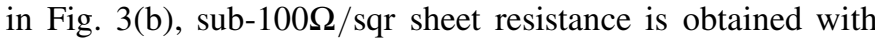
$>85 \%$ transmittance with 3 layers of printed PEDOT:PSS. The result shows the feasibility of using inkjet-printed PEDOT:PSS electrodes for flexible optoelectronic devices.

In terms of device fabrication, it is very important to balance the transmittance and resistance of the electrodes. For the bottom electrodes, we simply use the 8-layer films since there's no light illumination from the bottom and the resistance should be as low as possible to achieve efficient carrier transport. For the top layer where the optical transparency is important, we should consider the photoconductivity equation. According to ref. [13], we can write the photocurrent $I_{p}$ under bias voltage $V$ and optical illumination power $P_{o p t}$ as:

$$
I_{p}=q \eta \frac{P_{o p t}}{h v} \frac{\left(\mu_{n}+\mu_{p}\right) \tau}{t^{2}} T\left(V-I_{p} R\right)
$$

$\mu_{n}$ and $\mu_{p}$ are electron and hole mobility in PEDOT:PSS material, respectively. $h v$ is the illumination photon energy and $q$ is the electron charge. $\tau$ is the carrier lifetime. $t$ is the thickness of the NCQD thin film, and here it is roughly the thickness of the tracing paper but could be changed when compressing the structure. $\eta$ is the quantum efficiency of the NCQD thin film. R and T are the resistance and transmittance of the electrodes. We can solve the equation iteratively, or as a good approximation, we can ignore the voltage drop through the electrode, because of the relatively good conductance of the electrodes $(<150 \Omega /$ sqr for two or more layers) compared to the NCQD thin film $(\sim \mathrm{M} \Omega)$. Therefore, a linear dependence of the photocurrent on the light transmittance is a good approximation. In consideration of film uniformity, electrical conductance and optical transparency, we choose 3-layer PEDOT:PSS as the top electrodes.

\section{DeVice Performance}

A photodetector with an active device area of $5 \mathrm{~mm} \times 5 \mathrm{~mm}$ (Fig. 2) is characterized using a Cascade M150 low-noise probe station and a $532 \mathrm{~nm}$ continuous laser source with an intensity of $75 \mathrm{~mW} / \mathrm{cm}^{2}$ at a spot diameter of $2.44 \mathrm{~mm}$. About $75 \mu \mathrm{g}$ CdSe NCQDs are dispersed in the tracing paper and resulting in a density of $\sim 75 \mathrm{mg} / \mathrm{cm}^{3}$. Photoresponse of a flat device under different optical powers has been reported in [14]. In this letter, we characterized the device performance under bending radii of $4.8 \mathrm{~mm}, 5.5 \mathrm{~mm}, 6.5 \mathrm{~mm}$, and $9.2 \mathrm{~mm}$ (Fig. 4). With a decrease in bending radius, we find the response of the device increase both in dark current and total current under illumination [Fig. 4(a)]. We attribute the reason to that the bottom transparency film tends to have a smaller radius than that of the top film under bending, which squeezes the cellulose tracing paper. Hence the device with smaller bending radius tends to have thinner interlayer spacing. In view of the insulating nature of tracing paper, the conductivity of the NCQD film is mainly from the tunneling between the nano-particles, as typical NCQD devices. Therefore thinner spacing leads to better photoconductivity, as is also shown in Eq. (2). The net photoresponsivity for each bending radius is calculated and shows strong linear dependence on the bias voltage, which is in good agreement with typical photoconductor device performance. The fluctuation in the curve is mainly due to the unstable laser source. The relatively low response of the current device is due to the large interlayer spacing and scarce NCQD density in cellulose structure.

In order to improve the performance, an easy way is to clip the device between two stiff plates, which could be under bending, and we were able to obtain significantly better 

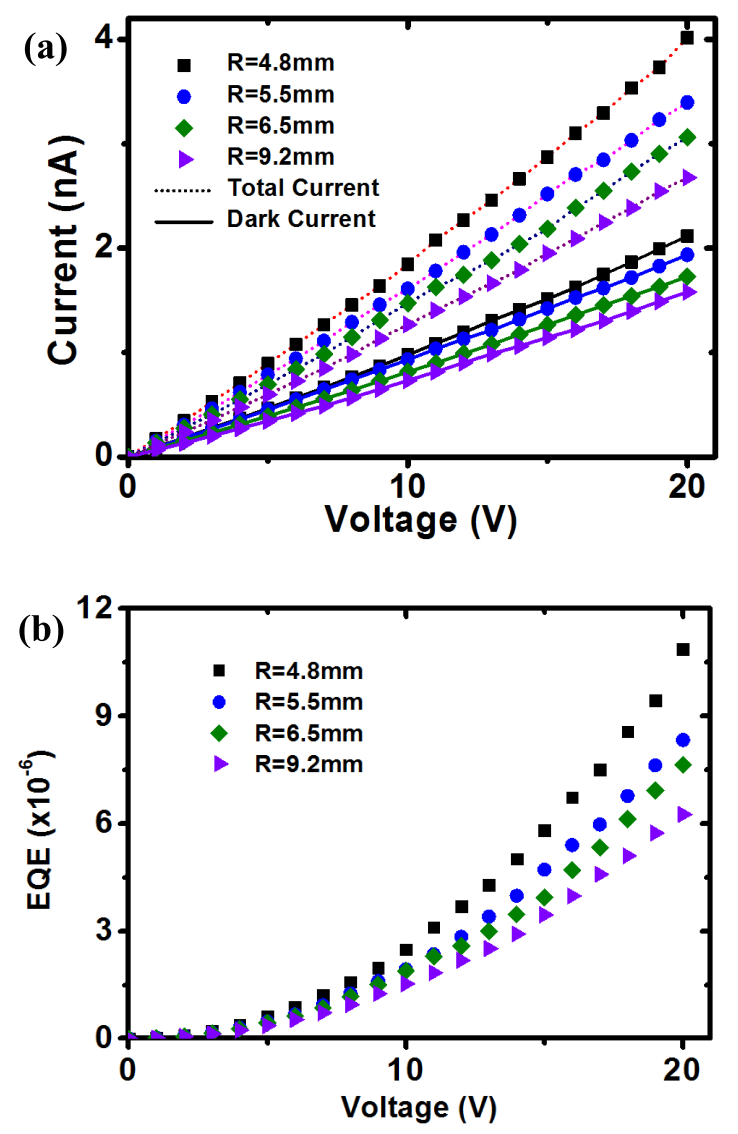

Fig. 4. (a) I-V characterizations of the device with bending radii of $4.8 \mathrm{~mm}$, $5.5 \mathrm{~mm}, 6.5 \mathrm{~mm}$, and $9.2 \mathrm{~mm}$, under $532 \mathrm{~nm}$ light illumination. (b) The external quantum efficiency (EQE) of the flexible NCQD photodetector vs. bias voltage at different bending radii.

results using this approach [14]. An alternate way is to use a thinner and more porous material as interspacing layer. We found bio-membranes, such as tree leaf, reed inner membrane, etc., have much thinner and more porous structures as well as better transparency. Higher photoresponsivity (>1\% EQE) has been found by applying these membranes in substitution of the tracing paper. More experiments and investigation are currently underway.

For applications in wearable flexible devices, the device should maintain its performance after many times of bending. We have performed this test on our flexible NCQD photodetector and found our device is able to maintain consistent performance after more than 100 times of bending. In terms of long-term reliability, we have seen little degradation in the performance of our devices after 2 months storage time. The sandwich structure helps to protect the device from degrading without further encapsulation process.

\section{CONClusion}

In summary, we successfully developed a simple and low-cost fabrication method and demonstrated a flexible threelayer thin-film NCQD photoconductive detector with inkjetprinted electrodes on transparency films. The printed polymer electrodes showed ITO-comparable conductivity and transmittance at visible wavelength. We were also able to form a continuous NCQD thin film on a tracing paper and use it as the active layer for photodetectors. Short-circuiting between the electrodes, a common challenge for vertical flexible devices, was prevented due to the insulating nature of the tracing paper. We reported photo-responsivity performance of the photodetectors. This process can be adapted to large-area, large-scale fabrication, and have potential impact in various optoelectronic applications such as photovoltaics, sensors, and displays.

\section{REFERENCES}

[1] L. Groenendaal, F. Jonas, D. Freitag, H. Pielartzik, and J. R. Reynolds, "Poly(3,4-ethylenedioxythiophene) and its derivatives: Past, present, and future," Adv. Mater., vol. 12, no. 7, pp. 481-494, 2000.

[2] Z. Chen, B. Cotterell, and W. Wang, "The fracture of brittle thin films on compliant substrates in flexible displays," Eng. Fract. Mech., vol. 69, no. 5, pp. 597-603, 2002

[3] C. Srichan, T. Saikrajang, T. Lomas, A. Jomphoak, T. Maturos, D. Phokaratkul, et al., "Inkjet printing PEDOT:PSS using desktop inkjet printer," in Proc. 6th Int. Conf. ECTI, 2009, pp. 465-468.

[4] S. Ummartyotin, J. Juntaro, C. Wu, M. Sain, and H. Manuspiya, "Deposition of PEDOT: PSS nanoparticles as a conductive microlayer anode in OLEDs device by desktop inkjet printer," $J$. Nanomater. vol. 2011, pp. 1-7, Apr. 2011.

[5] M. Böberl, M. V. Kovalenko, S. Gamerith, E. J. W. List, and W. Heiss, "Inkjet-printed nanocrystal photodetectors operating up to $3 \mu \mathrm{m}$ wavelengths," Adv. Mater, vol. 19, pp. 3574-3578, Nov. 2007.

[6] S. H. Ko, H. Pan, C. P. Grigoropoulos, C. K. Luscombe, J. M. J. Fréchet, and D. Poulikakos, "All-inkjet-printed flexible electronics fabrication on a polymer substrate by low-temperature high-resolution selective laser sintering of metal nanoparticles," Nanotechnology, vol. 18, no. 34 p. 345202, 2007.

[7] K. Kordás, et al., "Inkjet printing of electrically conductive patterns of carbon nanotubes," Small, vol. 2, nos. 8-9, pp. 1021-1025, 2006.

[8] Y. Yoshioka and G. E. Jabbour, "Desktop inkjet printer as a tool to print conducting polymers," Synth. Metals, vol. 156, pp. 779-783, Jun. 2006.

[9] M. C. Barr, et al., "Direct monolithic integration of organic photovoltaic circuits on unmodified paper," Adv. Mater., vol. 23, pp. 3499-3505, Aug. 2011.

[10] A. Manekkathodi, M.-Y. Lu, C. W. Wang, and L.-J. Chen, "Direct growth of aligned zinc oxide nanorods on paper substrates for low-cost flexible electronics," Adv. Mater, vol. 22, no. 36, pp. 4059-4063, 2010.

[11] C.-J. Lee, D.-G. Moon, and J.-I. Han, "29.2: Top emission organic el display on paper substrate," SID Symp. Dig. Tech. Papers, vol. 35, no. 1, pp. 1005-1007, 2004.

[12] L. Hu, D. S. Hecht, and G. Grüner, "Percolation in transparent and conducting carbon nanotube networks," Nano Lett., vol. 4, no. 12, pp. 2513-2517, 2004.

[13] S. M. Sze and K. K. Ng, Physics of Semiconductor Devices, 3rd ed. Hoboken, NJ, USA: Wiley, 2007.

[14] J. Wu and L. Y. Lin, "Flexible thin-film nanocrystal quantum dot photodetectors on unmodified transparency films," in Proc. IPC, 2012, pp. 380-381. 